\title{
A novel mutation in the interleukin-1 receptor antagonist associated with intrauterine disease onset
}

\author{
Ender Altiok ${ }^{\mathrm{a}, 1}{ }$, Figen Aksoy ${ }^{\mathrm{b}}$, Yıldız Perk ${ }^{\mathrm{c}}$, Fulya Taylan ${ }^{\mathrm{d}, 1}$, \\ Peter W. Kim ${ }^{\mathrm{e}}$, Barbaros Ilıkkan ${ }^{\mathrm{c}}$, Gülten Turkkani Asal ${ }^{\mathrm{f}}$, \\ Raphaela Goldbach-Mansky ${ }^{\mathrm{e}}$, Ozden Sanal ${ }^{\mathrm{f}, *}$
}

\footnotetext{
a Department of Medical Biology, Acibadem University Faculty of Medicine, Istanbul, Turkey

b Department of Pathology, Cerrahpasa Medical School, Istanbul, Turkey

c Neonatology Unit, Cerrahpașa Medical School, İstanbul, Turkey

d Acibadem Genetika, Istanbul, Turkey

e National Institute of Arthritis and Musculoskeletal and Skin Diseases, NIH, Bethesda, MD, USA

${ }^{f}$ Division of Pediatric Immunology, Hacettepe University Medical School, Ankara, Turkey
}

Received 30 May 2012; accepted with revision 7 August 2012

Available online 16 August 2012

\section{KEYWORDS}

Autoinflammatory syndrome;

Deficiency of interleukin-

1 receptor antagonist;

Intrauterine onset;

Immunodeficiency

\begin{abstract}
Deficiency of the IL-1 receptor antagonist (DIRA) is a recently described rare autoinflammatory disease, caused by loss of function mutations in IL1RN leading to the unopposed activation of the IL-1 pathway. We describe a novel nonsense mutation in the IL1RN gene, associated with early intrauterine onset, death and multiorgan involvement in a prematurely born baby. The protein prediction model indicated that the novel Q119X mutation would result in a nonfunctional protein by impairing the ability of the IL-1Ra to bind and antagonize signaling through the IL-1R. Since the disorder may mimic severe bacterial infections and the treatment with anakinra is life saving, we intend to raise awareness of the syndrome and the possibility of a founder mutation that may lead to the diagnosis of additional cases in Turkey. The clinical suspicion of DIRA is critical to avoid improper management of the patients with antibiotics alone and death from multiorgan failure.
\end{abstract}

(c) 2012 Elsevier Inc. All rights reserved.

\footnotetext{
* Corresponding author at: Division of Pediatric Immunology, Hacettepe University Medical School, Ankara 06100, Turkey. Fax: +90 312 2959969.

E-mail addresses: altioke@gmail.com (E. Altiok), ctfpatoloji@gmail.com (F. Aksoy), perky@istanbul.edu.tr (Y. Perk), fulyataylan@gmail.com (F. Taylan), kimp@mail.nih.gov (P.W. Kim), goldbacr@mail.nih.gov (R. Goldbach-Mansky), sanaloz@gmail.com (0. Sanal).

${ }^{1}$ Present address: Department of Genetics and Bioengineering, Okan University, Tuzla, Istanbul 34959, Turkey.
} 


\section{Introduction}

Autoinflammatory syndromes are inherited diseases characterized by recurrent episodic systemic inflammation often presenting with cutaneous and rheumatologic manifestations [1-5]. These diseases are caused by dysregulated activation of inflammasomes, which are critical for the activation of the proinflammatory cytokine interleukin (IL)-1 $\beta$, a powerful mediator of inflammatory responses [6-8]. Interleukin 1 receptor antagonist (IL1Ra), the first endogenous cytokine receptor antagonist described [9] inhibits the activities of IL-1 $\alpha$ and IL-1 $\beta$. Recently, a severe autoinflammatory condition presenting with neonatal-onset disease and clinical similarities to NOMID, was found to be caused by homozygous mutations in IL1RN $[10,11]$. It has been named as deficiency of the IL-1 receptor antagonist (DIRA). Mutations in IL1RN lead to complete absence [10,11] or dysfunction [12] of IL-1Ra protein and thus to unopposed action of IL-1 alpha and beta on the IL- 1 receptors due to the lack of the competitive antagonist, IL-1Ra. The disease therefore is a model of the inability to downregulate and stop the IL-1 "danger response", and the severe inflammatory reaction seen in these patients can resemble an acute severe systemic infection. If left untreated, the disease can lead to death from multiple organ failures. If recognized early, the treatment with replacement of the deficient protein, anakinra, the recombinant human IL-1RA, induces rapid and complete remission [10-13].

In this report, we describe two affected children from the same family; with a severe DIRA phenotype one of the patients presents with early onset intrauterine disease and intrauterine demise, and the second infant was prematurely born at week 31 and within a week of birth presented with inflammatory symptoms that were considered as sepsis, pyodermia and immunodeficiency. He did not respond to antimicrobial agents, developed hemophagocytosis and died at the age of 4 months.

\section{Methods}

\subsection{Subjects}

The first pregnancy of a consanguineous couple (3rd degree relatives) resulted in an empty embryonic sac and was terminated by curettage.

\subsubsection{Case 1}

The second pregnancy in 2004 had resulted in an intrauterine demise at 27 weeks of gestation. During a follow-up examination an ultrasonogram (USG) performed at 23 weeks of gestation estimated the gestational age at 20 weeks, and showed edema on areas of the scalp and skin, minimal intraabdominal ascites, short ribs, narrow thorax and polyhydroamnios. An amniocentesis was done and studies including chromosomal analysis did not reveal a specific diagnosis. The fetus (female) died at 27 weeks of gestation and was medically aborted. The weight was $1100 \mathrm{~g}$, head and chest circumferences were 24 and $23 \mathrm{~cm}$ respectively. There was no inflammation of the umbilical cord. The features of the postmortem X-ray examination of the bones were interpreted as osteomyelitis (pictures are not available). The postmortem histopathological examination showed abscesses with dense neutrophilic infiltrates and extensive tissue destruction in the thymus, adrenal gland and myocardium (Fig. 1). The morphological features of the probable colonies of fungal hyphae within a neutrophilic abscess were noted in the pericardium and adrenal tissue, however, no microorganisms were grown from the samples obtained from the myocardium and fungal colonies were not present in other affected tissues.

\subsubsection{Case 2}

The third pregnancy produced a live male infant, born at 31 weeks gestational age in 2005 . The infant was admitted to the newborn intensive care unit (NICU) because of respiratory distress. During the first week of life he developed pustular lesions on the scalp and extremities and CRP was noted to be elevated. He was started on the antibiotics, teicoplanin, amikacin, and liposomal amphotericin B and was referred to another hospital with the diagnosis of primary immunodeficiency disease at the age of 17 days. Physical examination on admission showed pustular skin lesions on the scalp, extremities, right elbow, right ankle, left knee and lumbar area that were considered to be infectious, he also had pretibial edema. Bacterial and fungal cultures from blood and skin did not grow out any microorganisms. An immunological workup including NBT, serum lgs, absolute lymphocyte count and lymphocyte subsets was normal. Skin lesions responded somewhat to cefazolin, amikacin, fucidine and zinc therapy. He was discharged from the hospital at the age of 36 days. Seven days after discharge, he was re-admitted to the hospital with dyspnea and cyanosis during feeding. The physical examination revealed bilateral pretibial edema, rales on both lung fields, and swelling of the right elbow and wrist. The results of the laboratory workup showed leukocytosis (WBC count varied between 23,000 and $61,000 / \mathrm{mm}^{3}$ ) with increased percentage of neutrophils and bands and increased CRP (100->160 mg/L, reference value being $<8 \mathrm{mg} / \mathrm{L}$ ). Pulmonary $\mathrm{X}$-ray showed bilateral diffuse infiltrates. Skeletal X-rays showed multifocal osteomyelitis of bilateral femoral heads (pictures are not available); however, a bone biopsy was not done. His general status and respiratory distress worsened progressively and mechanical ventilation was started. Blood cultures obtained on that admission remained negative for microorganisms. Antifungal and broad spectrum antibiotic treatment was started because of concerns of disseminated sepsis with pneumonia, osteomyelitis and septic arthritis. Despite the therapy no improvement was observed in skin lesions. Hemophagocytosis was demonstrated in bone marrow aspiration smear. CMV was found in peripheral blood $(11,100$ copy $/ \mathrm{mL})$. IVIG, dexamethasone and gangcyclovir were added to the therapy due to hemophagocytosis and CMV infection. Respiratory distress worsened progressively and the patient died at 4 months of age. No suitable material from the diseased patient was available for further studies. The couple had no family history of autoimmunity, inflammatory skin or bone disorder.

\subsection{Genetic and structural analysis}

Based on the clinical similarities between our patients with the other DIRA patients described in the literature, we hypothesized that IL1RN mutations could be the cause for the disease in our patients. We therefore analyzed the IL1RN 

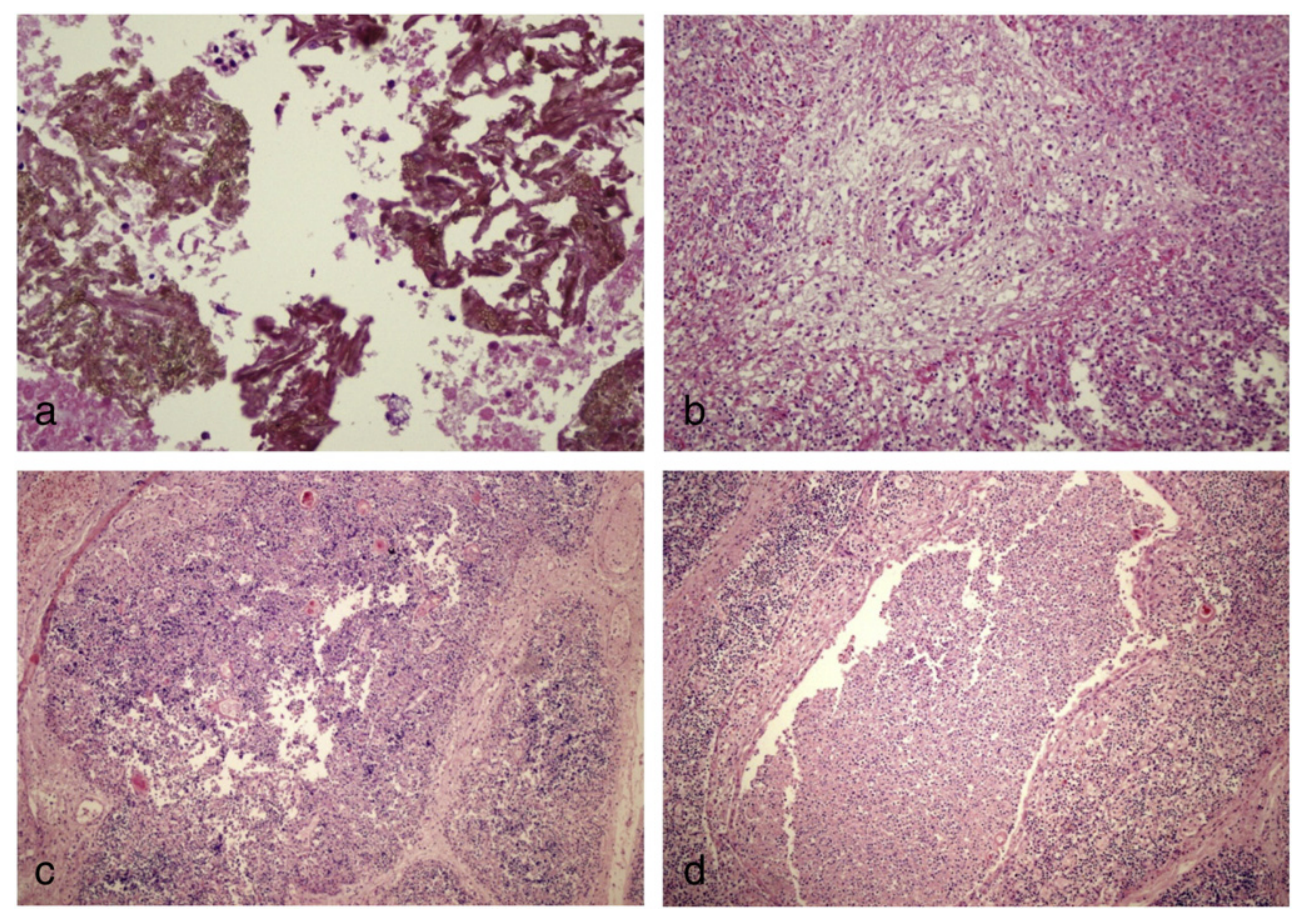

Figure 1 Histopathological features of the sibling who died intrauterine at 20 weeks of gestation. a: Adrenal gland: dense neutrophilic infiltrate with tissue destruction, colonies of fungal hyphae within neutrophilic abscesses; b: myocardium: heavy infiltrate of neutrophils which destroyed muscle fibers in some areas, mixed inflammatory cell infiltration in an arterial wall and periarterial area; c,d: thymus: Absence of cortico-medullary demarcation. Cortex was poor of lymphocytes (lymphoid cell depletion), aggregates of PMNs, lymphocyte, and histiocytes with tissue destruction.

gene sequence from the parents' blood samples, as no suitable materials from the affected patients were available. Genomic DNA extracted from peripheral blood of the parents has been amplified by PCR for coding exons of the IL1RN gene using primers flanking both sides of the corresponding exon (primer sequences and PCR conditions are available upon request). Following purification of $5 \mu \mathrm{L}$ aliquots of PCR amplicons by ExOSAP-IT treatment, cycle sequencing reaction was performed by using a DYEnamic ET Terminator Cycle Sequencing Kit (GE Healthcare). After dye cleaning, the products were electrophoresed and analyzed in the MegaBACE 1000 Genetic Analyzer. The reference mRNA and protein sequences for the IL1RN gene were NM_173842.2 and NP_776214.1, respectively.

The effect of the mutation on the protein structure and function has been studied by a prediction model as described previously [14]. The structure of IL-1Ra bound to IL-1RI has been taken as a template for this prediction [14].

\section{Results and discussion}

We describe the clinical and laboratory features of 2 siblings, an in utero demise fetus at twenty seven weeks of gestation with inflammatory features suggestive of intrauterine onset of DIRA, and an infant with clinical manifestations of DIRA born at 31 weeks of gestation. The DNA from the affected fetus and the newborn was not available; however, the sequencing analysis of the coding exons of IL1RN gene of the parents revealed that they were heterozygous carriers of a nonsense mutation c355C > T (p.Q119X) on exon-4 of the IL1RN gene. Substitution of $\mathrm{a} \mathrm{C}$ to $\mathrm{a} \mathrm{T}$ at position 355 of the reference cDNA has created a premature TAG stop codon expected to result in a truncated IL-1Ra by early termination of the protein. The prediction model indicated that the Q119X mutation would alter IL-1Ra binding to IL-1RI receptor by two mechanisms (Fig. 2). First, IL1-RI binding is impaired because the Q119X mutant form of IL-1Ra loses amino acids (A152, D153, Y172, E175) that bind directly to IL-1RI. Second, the $\beta$-barrel of IL-1Ra is unable to fold properly since the $\beta 8-, \beta 9-$ and $\beta 12$-strands are missing; this likely also disrupts folding for the remainder of IL-1Ra. Together, these likely effects predict a nonfunctional protein by impairing the ability of Q119X mutant IL-1Ra to bind and antagonize IL-1RI.

The Q119X mutation is a novel mutation associated with DIRA. Our data suggest that in some cases the inflammatory manifestations can start in utero and lead to intrauterine death.

DIRA is a rare condition that usually presents during the neonatal period. DIRA patients present with systemic inflammation, respiratory distress, joint swelling, pustular rash, multifocal osteomyelitis and periosteitis typically affecting the distal ribs and the long bones [10-13]. Other clinical features include heterotopic bone formation around the proximal femur, thrombosis, rarely vasculitis and non-union of the odontoid [10-13]. The phenotype of our patients presented here includes respiratory insufficiency, pustulosis, arthritis, leukocytosis, and persistently elevated levels of CRP and erythrocyte sedimentation rate without fever. The clinical and laboratory features in the live born infant, the product of the third pregnancy, and the aborted fetus, the product of the second pregnancy were 


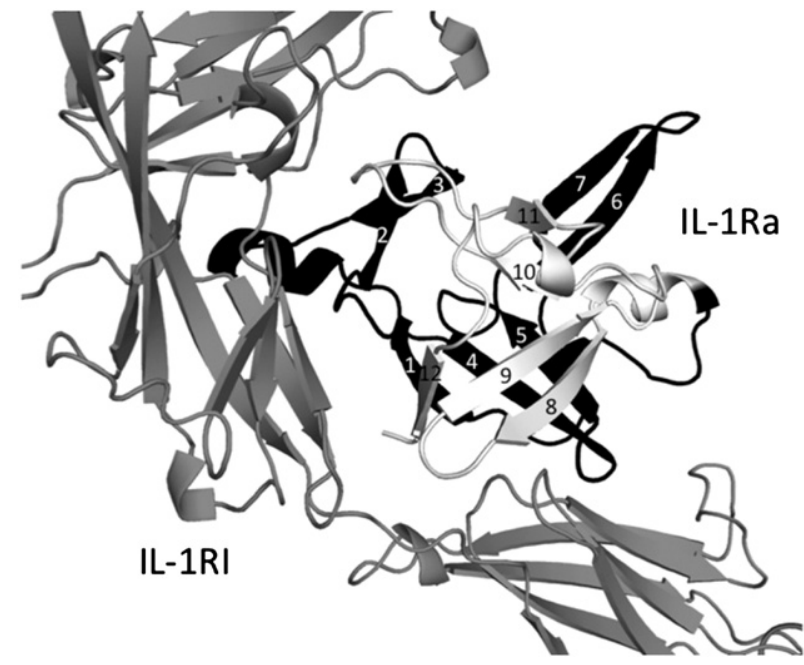

Figure 2 Prediction model for the effect of p.Q119X mutation on IL-1Ra. Structure of IL-1Ra bound to IL-1RI (Schreuder et al., Nature $386: 194-200,1997)$ is taken as a template. IL-1Ra has twelve $\beta$-strands with strands $\beta 1, \beta 4, \beta 5, \beta 8, \beta 9$ and $\beta 12$ forming a six-stranded beta-barrel. Missing amino acids in the Q119X mutant form of IL-1Ra are shown in white, and the amino acids that are present are shown in black.

reminiscent of DIRA. All reported patients with DIRA presented at birth or by 2.5 weeks of age. While clinical symptoms develop post partum, one third of the reported patients were born prematurely, between 33-38 weeks and 31 weeks in our live born patient, and/or small for gestational age (SGA) and/ or with minimal clinical symptoms suggesting the possibility of intrauterine disease onset, however no case demonstrating intrauterine onset has been reported so far. It has been hypothesized that the high concentrations of IL-1Ra in the amniotic fluid might be protective in utero and that the triggers of the inflammation such as skin trauma and exposure to organisms may be limited in utero, and more likely occur post partum $[10,15]$. IL-1 has previously been implicated in premature births $[15,16]$, but whether there is an independent link between the premature birth and IL-1 concentrations is currently unknown.

In this report we have described morphological and histopathological features of DIRA in the fetus aborted in utero at 27 weeks of gestation. We observed necrotizing abscess formation in the thymus, surrenal gland and the myocardium. The presence of colonies of fungal hyphae within the neutrophilic abscesses may suggest that a transvaginal fungal infection might have triggered the earlier onset of the disease in the fetus. However, no microorganisms were grown from the samples obtained from the myocardium and fungal colonies were not present in these tissues suggesting that a contamination might have happened after the in utero cell death. Besides, characteristics of intrauterine fungal infection (chorioamnionitis and funisitis, infective alveolitis or gastrointestinal involvement) were not present. Neutrophil infiltration in various organs, findings of fetal USG at 23rd week of gestation, edema of the scalp and skin, minimal intraabdominal ascites, short ribs, narrow thorax and polyhydromnios, and the X-ray bone findings were consistent with previous reports on early post partum manifestations of DIRA. It has not been possible to access the fetal material for genetic analyses. The novel mutation, Q119X found as a heterozygous mutation in both parents and likely constitutes the cause of the disease in the children. Whether this mutation in general causes a very severe phenotype leading even to intrauterine death, or whether the severe phenotype is just seen in this family needs to be shown in other cases with this mutation.

To date, 16 children with DIRA have been reported, nine by Aksentijevich et al. [10]; one from Newfoundland, five from three families from Holland, and two brothers from Lebanon were homozygous for point mutations in the IL1RN, and one child from Puerto Rico was homozygous for a $175 \mathrm{~kb}$ genomic deletion that includes IL1RN and five adjacent genes. Four additional patients with $175 \mathrm{~kb}$ deletion were reported [11,17-19]. Recently, a male infant with a compound heterozygous mutation was described [13]. While these mutations were shown to lead to absence of the protein, a recent mutation in 2 patients from Brazil showed a 15 bp inframe deletion that led to the expression of a non-functional protein and a clinical phenotype consistent with the clinical phenotype of other DIRA patients (12). The Q119X mutation is novel and although the parents are consanguineous, there is a possibility that this mutation is a founder mutation in Turkey. More work is needed to determine the geographic area and frequency of this mutation. The awareness of this mutation in the respective population could allow for prenatal screening.

Since the clinical presentation of DIRA early in life resembles neonatal sepsis, presenting with infectious osteomyelitis and systemic inflammation as in our case and the treatment with anakinra is life saving, a clinical suspicion of DIRA is critical to avoid improper management of the patients with antibiotics alone and death from multiorgan failure [1,10-13,17-19]. Thus treatment with anakinra should be considered as soon as clinical suspicion for an autoinflammatory condition exists even before a definitive diagnosis is made by genetic analysis.

\section{Conflict of interest statement}

The authors declare that there are no conflicts of interest.

\section{References}

[1] R. Goldbach Mansky, Immunology in clinic review series; focus on autoinflammatory diseases: update on monogenic autoinflammatory diseases: the role of interleukin (IL)-1 and an emerging role for cytokines beyond IL-1, Clin. Exp. Immunol. 167 (2011) 391-404.

[2] V.U. Ozkurede, L. Franchi, Immunology in clinic review series; focus on autoinflammatory diseases: role of inflammasomes in autoinflammatory syndromes, Clin. Exp. Immunol. 167 (2011) $382-390$.

[3] I. Touitou, I. Kone-Paut, Autoinflammatory diseases, Best Pract. Res. Clin. Rheumatol. 22 (2008) 811-829.

[4] K.M. Hull, N. Shoham, J.J. Chae, I. Aksentijevich, D.L. Kastner, The expanding spectrum of systemic autoinflammatory disorders and their rheumatic manifestations, Curr. Opin. Rheumatol. 15 (2003) 61-69.

[5] M. Braun-Falco, T. Ruzicka, Skin manifestations in autoinflammatory syndromes, J. Dtsch. Dermatol. Ges. 9 (2011) 232-245.

[6] C. Gabay, C. Lamacchia, G. Palmer, IL-1 pathways in inflammation and human diseases, Nat. Rev. Rheumatol. 6 (2010) 232-241. 
[7] H.M. Hoffman, A.A. Wanderer, Inflammasome and IL-1betamediated disorders, Curr. Allergy Asthma Rep. 10 (2010) 229-235.

[8] O. Gross, C.J. Thomas, G. Guarda, J. Tschopp, The inflammasome: an integrated view, Immunol. Rev. 243 (2011) 136-151.

[9] D.B. Carter, M.R. Deibel Jr., C.J. Dunn, C.S.C. Tomich, A.L. Laborde, J.L. Slightom, A.E. Berger, M.J. Bienkowski, F.F. Sun, R.N. McEwan, et al., Purification, cloning, expression and biological characterization of an interleukin-1 receptor antagonist protein, Nature 344 (1990) 633-638.

[10] I. Aksentijevich, S.L. Masters, P.J. Ferguson, P. Dancey, J. Frenkel, A. van Royen-Kerkhoff, R. Laxer, U. Tedgård, E.W. Cowen, T.H. Pham, et al., An autoinflammatory disease with deficiency of the interleukin-1-receptor antagonist, N. Engl. J. Med. 360 (2009) 2426-2437.

[11] S. Reddy, S. Jia, R. Geoffrey, R. Lorier, M. Suchi, U. Broeckel, M.J. Hessner, J. Verbsky, An autoinflammatory disease due to homozygous deletion of the IL1RN locus, N. Engl. J. Med. 360 (2009) 2438-2444.

[12] A.A. Jesus, M. Osman, C.A. Silva, P.W. Kim, T.H. Pham, M. Gadina, B. Yang, D.R. Bertola, M. Carneiro-Sampaio, P.J. Ferguson, et al., A novel mutation of IL1RN in the deficiency of interleukin-1 receptor antagonist syndrome: description of two unrelated cases from Brazil, Arthritis Rheum. 63 (2011) 4007-4017.

[13] M. Stenerson, K. Dufendach, I. Aksentijevich, J. Brady, J. Austin, A.M. Reed, The first case of compound heterozygous IL1RN mutations causing deficiency of the interleukin-1-receptor antagonist, Arthritis Rheum. 63 (2011) 4018-4022.
[14] H. Schreuder, C. Tardif, S. Trump-Kallmeyer, A. Soffientini, E. Sarubbi, A. Akeson, T. Bowlin, S. Yanofsky, R.W. Barrett, A new cytokine-receptor binding mode revealed by the crystal structure of the IL-1 receptor with an antagonist, Nature 386 (1997) 194-200.

[15] S.S. Chow, M.E. Craig, C.A. Jones, B. Hall, J. Catteau, A.R. Lloyd, W.D. Rawlinson, Differences in amniotic fluid and maternal serum cytokine levels in early midtrimester women without evidence of infection, Cytokine 44 (2008) 78-84.

[16] R. Romero, F. Gotsch, B. Pineles, J.P. Kusanovic, Inflammation in pregnancy: its roles in reproductive physiology, obstetrical complications, and fetal injury, Nutr. Rev. 65 (2007) 194-202.

[17] K. Minkis, I. Aksentijevich, R. Goldbach-Mansky, C. Magro, R. Scott, J.G. Davis, N. Sardana, R. Herzog, Interleukin 1 receptor antagonist deficiency presenting as infantile pustulosis mimicking infantile pustular, psoriasis, Arch. Dermatol. 148 (2012) 747-752.

[18] C.N. Brau-Javier, J. Gonzales-Chavez, J.R. Toro, Chronic cutaneous pustulosis due to a $175-k b$ deletion on chromosome 2q13: excellent response to anakinra, Arch. Dermatol. 148 (2012) 301-304.

[19] C. Schnellbacher, G. Ciocca, R. Menendez, I. Aksentijevichl, R. Goldbach-Mansky, A.M. Duarte, R. Rivas-Chacon, Deficiency of interleukin-1 receptor antagonist responsive to anakinra, Pediatr. Dermatol. (2012), http://dx.doi.org/10.1111/j.1525-1470.2012. 01725.x. 